\title{
Transganglionic Transport of HRP from the Circumvallate Papilla of the Rat
}

\author{
ROBERT M. BRADLEY ${ }^{1,2}$, CHARLOTTE M. MISTRETTA ${ }^{1,3,4}$, CAROLYN A. BATES $^{5}$ and \\ HERBERT P. KILLACKEY 5 \\ 'Department of Oral Biology, University of Michigan, School of Dentistry, ${ }^{2}$ Department of Physiology, School of Medicine, \\ ${ }^{3}$ Center for Nursing Research, School of Nursing, ${ }^{4}$ Center for Human Growth and Development, Ann Arbor, MI 48109 and \\ ${ }^{5}$ Department of Psychobiology University of California at Irvine, Irvine, CA 92717 (U.S.A.)
}

(Accepted April 16th, 1985)

Key words: circumvallate papilla - glossopharyngeal nerve — solitary tract and nucleus - taste — von Ebner's gland — inferior salivatory nucleus - transganglionic transport - taste bud

\begin{abstract}
To learn whether horseradish peroxidase (HRP) injections in gustatory papillae on the tongue can be used to study central topographical projections of taste buds and papillae, injections were made into the circumvallate papilla in rats. Labeled central projections after papilla injections were compared to projections after applying HRP to the cut glossopharyngeal nerve. Papilla injections result in HRP transport by afferent and efferent fibers of the glossopharyngeal nerve, and the pattern of central projections is similar to that after labeling the cut nerve. Projections include a separation in the brainstem of afferent, dorsally located fibers and efferent, ventrally located fibers. Afferent fibers project to the solitary nucleus and the trigeminal system. Efferent projections label muscle motorneurons in the nucleus ambiguus and the cells of origin of parasympathetic preganglionic fibers, which form the inferior salivatory nucleus. The parasympathetic neurons labeled after papilla projections are preganglionic fibers to Remak's ganglia in the tongue; postganglionic fibers of these ganglia are the secretomotor supply to the von Ebner's glands. In summary, injections of HRP into gustatory papillae reliably label central projections of the papilla and can be used for studies to discern topography in central projections of the taste system. Injections into the circumvallate papilla also have demonstrated that the parasympathetic neurons innervating von Ebner's glands are located in the inferior salivatory nucleus.
\end{abstract}

\section{INTRODUCTION}

Horseradish peroxidase (HRP) has been used to trace the central connections of many sensory receptors, both by application to cut nerve afferents and by direct injection at the site of the receptor ${ }^{11}$. With the latter method, the injection site can be localized to specific areas and therefore, it is possible to determine whether peripheral receptors are represented topographically in the central nervous system ${ }^{1}$. Central connections of the taste system have been traced only after applying HRP to the cut central ends of gustatory nerves $6,8,15-18,22$. To begin studies of possible topographical projections from taste papillae to the central nervous system, we have injected HRP into the circumvallate papilla of the rat and compared the labeled central projections after papilla injection and after applying HRP to the cut glossopha- ryngeal nerve, which innervates the papilla and its taste buds. These experiments demonstrate the validity and usefulness of papilla injection for tracing central connections in the gustatory system.

\section{MATERIALS AND METHODS}

\section{Surgery}

Papilla injections were made in 18 male SpragueDawley rats weiging $30-160 \mathrm{~g}$ (aged 15-45 days). The rats were anesthetized with an intramuscular injection of a 1:1 mixture of Rompun ( $2 \mathrm{mg} / \mathrm{kg} \mathrm{b}$.wt.) and ketamine $(10 \mathrm{mg} / \mathrm{kg})$. For surgery, the rat was secured in a head holder and the cheek tissue cut from the angle of the mouth to the ascending ramus of the mandible. The lower jaw was retracted and the tongue depressed. Using a dissecting microscope the single circumvallate papilla was visualized (Fig. 1)

Correspondence: R.M. Bradley, Department of Oral Biology, School of Dentistry, University of Michigan, Ann Arbor, MI 48109, U.S.A. 
$A$
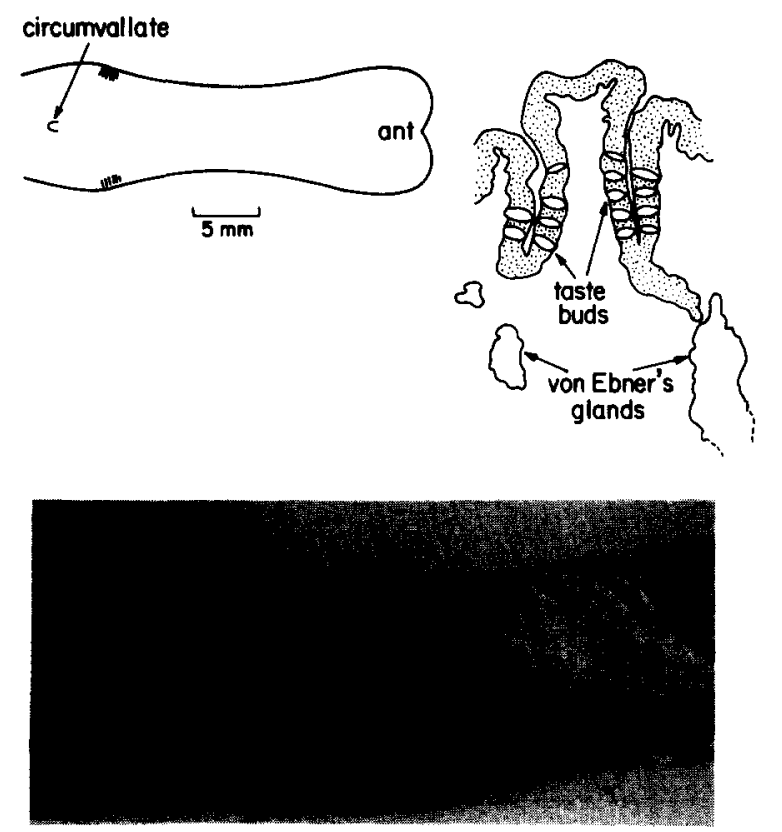

Fig. 1. A: diagram of the dorsal surface of the rat tongue to show the site of injection into the circumvallate papilla. B: diagrammatic sagittal section through the circumvallate papilla to show the position of the taste buds and von Ebner's glands. C: light-field photomicrograph of a coronal section through the circumvallate papilla to illustrate the reaction product produced at the injection site. The circumvallate papilla is located in the middle of the dorsal tongue epithelium, at the top of the figure. $\times 8$.

and $0.5-1.0 \mu \mathrm{l}$ of either a 5 or $10 \%$ solution of wheat germ agglutinin HRP (Sigma) was injected into the papilla from a Hamilton syringe. The cheek incisions were then sutured and the animal allowed to recover.

The central projections of the cut glossopharyngeal nerve (IX) were studied in 6 rats, for comparison with results from papilla injections. The anesthetized rat was placed supine and the cervical trunk of the IXth nerve was dissected free of surrounding tissue and cut. The central end of the nerve was placed on a piece of Parafilm and HRP crystals (Sigma VI) were applied. After 30 min the Parafilm was closed around the HRP and nerve end, and covered in vaseline. The incision was then sutured.

\section{Histochemistry}

After $48 \mathrm{~h}$ survival, the rats were anesthetized with sodium pentobarbital $(50 \mathrm{mg} / \mathrm{kg}$ b.wt.) and perfused transcardially. The blood was flushed from the vascu- lar system with $0.1 \mathrm{M}$ phosphate buffer containing $0.9 \%$ sodium chloride, $3 \%$ procaine $\mathrm{HCl}$ and $0.003 \%$ heparin, followed by a solution of $4 \%$ glutaraldehyde and $1 \%$ paraformaldehyde in $0.1 \mathrm{M}$ phosphate buffer ( $\mathrm{pH} 7.30$ ), and finally by cold $10 \%$ sucrose in $0.1 \mathrm{M}$ phosphate buffer. The brain and tongue were removed and stored for $24 \mathrm{~h}$ in cold $10 \%$ sucrose in $0.1 \mathrm{M}$ phosphate buffer. Serial, $40 \mu \mathrm{m}$, frozen sections of the brainstem, cervical spinal cord and posterior tongue were cut in the coronal plane on a sliding microtome. The tissue was treated with tetramethyl benzidine (TMB) as a chromagen for the demonstration of HRP reaction product ${ }^{11}$. Sections were mounted out of cold $0.1 \mathrm{M}$ acetate buffer ( $\mathrm{pH} 3.30$ ) onto chrome-alum-subbed slides. All sections were examined under dark-field and polarizing light microscopy 7 . The distribution of HRP label in the brainstem was plotted onto outlined sections of the brainstem using a drawing tube attached to the microscope. Selected sections were photographed.

\section{RESULTS}

\section{Injection site}

Examination of injected papillae showed that the HRP was confined to the area of the circumvallate papilla (Fig. 1C). The reaction product extended 2-3 $\mathrm{mm}$ below the papilla and could be traced $1-2 \mathrm{~mm}$ circumferentially beyond the papilla. The only cranial nerves in this site are the branch of the glossopharyngeal nerve that supplies the circumvallate papilla and the hypoglossal nerve branch that supplies the muscles below the papilla.

\section{Central projections of injected circumvallate papilla}

Since HRP was injected into the circumvallate papilla, which is located on the midline and innervated by both glossopharyngeal nerves, all brain structures were labeled bilaterally. HRP was transported retrogradely to the hypoglossal nucleus (XII) via motor endings in the tongue muscles. This central distribution of XII nerve fibers was not studied in detail, but has been described by Krammer et al. 10 .

HRP was transported transganglionically from the tongue to the brainstem via glossopharyngeal nerve fibers. Afferent fibers entered the brainstem over a distance of $0.8 \mathrm{~mm}$ that extended rostrally to the level of the dorsal cochlear nucleus $(0.4 \mathrm{~mm}$ rostral to its 

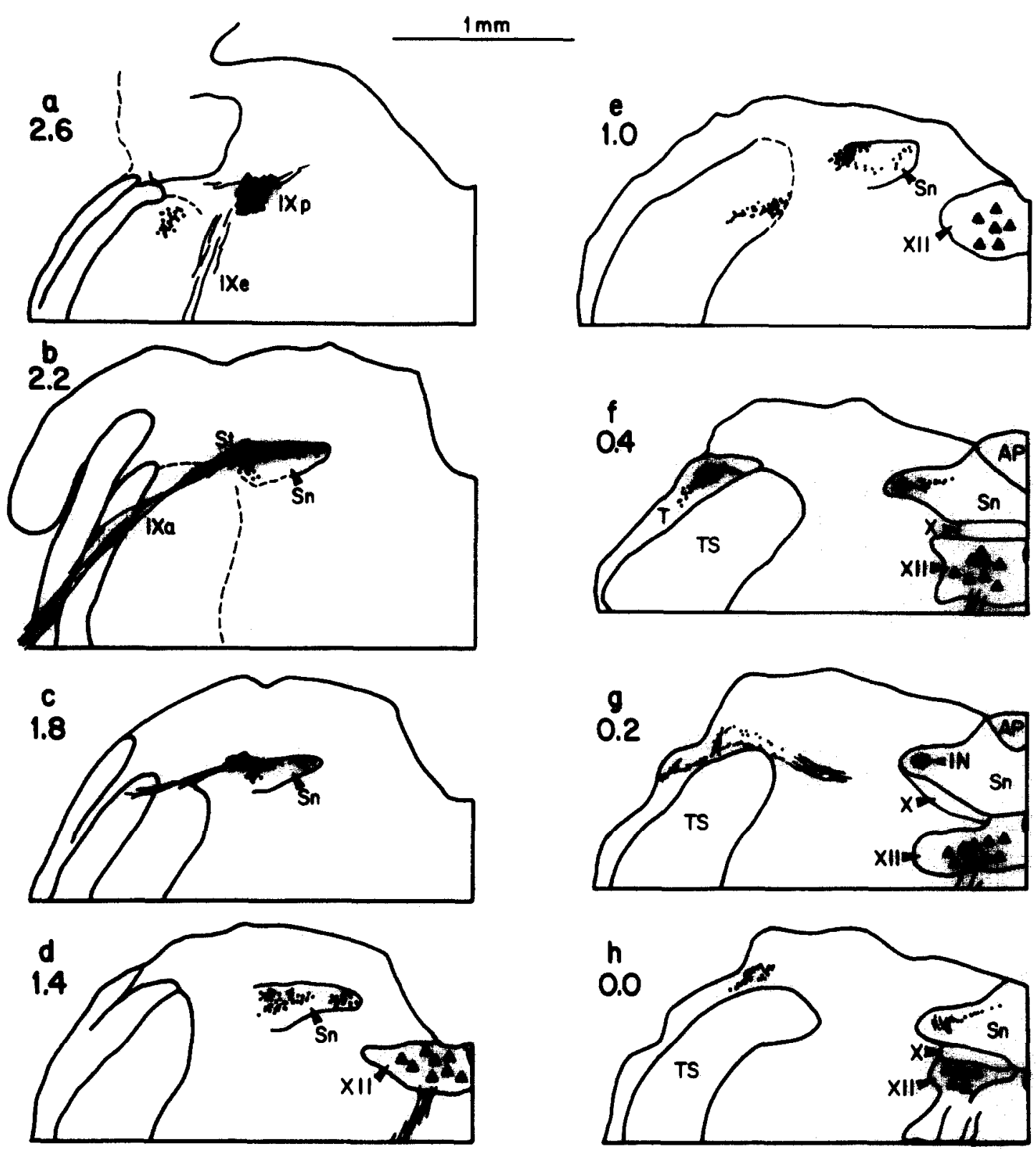

Fig. 2. Tracings of coronal sections through the brainstem of a rat in which HRP was injected into the circumvallate papilla. Only one side of the brain has been traced since the label was bilaterally symmetrical. In all cross sections, dots represent regions of axon terminals, irregular short lines represent fibers and fascicles of fibers, and triangles represent cells labeled after retrograde transport. AP, area postrema; IN, interstitial nucleus of the solitary tract; $\mathrm{Sn}$, solitary nucleus; $\mathrm{T}$, spinal tract of trigeminal complex; TS, spinal nucleus of trigeminal complex; IXa, glossopharyngeal afferent fibers; IXe, glossopharyngeal efferent fibers; IXp, parasympathetic cells of glossopharyngeal nerve; $\mathrm{X}$, dorsal motor nucleus of the vagus nerve; XII, hypoglossal nucleus. Numbers indicate approximate distance in mm rostral to the obex.

caudal border) and caudally to the rostral border of the XII motor nucleus (Fig. $2 \mathrm{a}-\mathrm{c}$ ). Within the brainstem the afferents joined both the spinal trigeminal tract (T) and the solitary tract (St) (Fig. 2b, f).

Labeled fascicles in the spinal trigeminal tract had both descending and ascending components. The ascending trigeminal component was traced as far as the genu of the facial nerve. However, the majority of fibers descended in the spinal trigeminal tract and became most dense at the level of the area postrema 


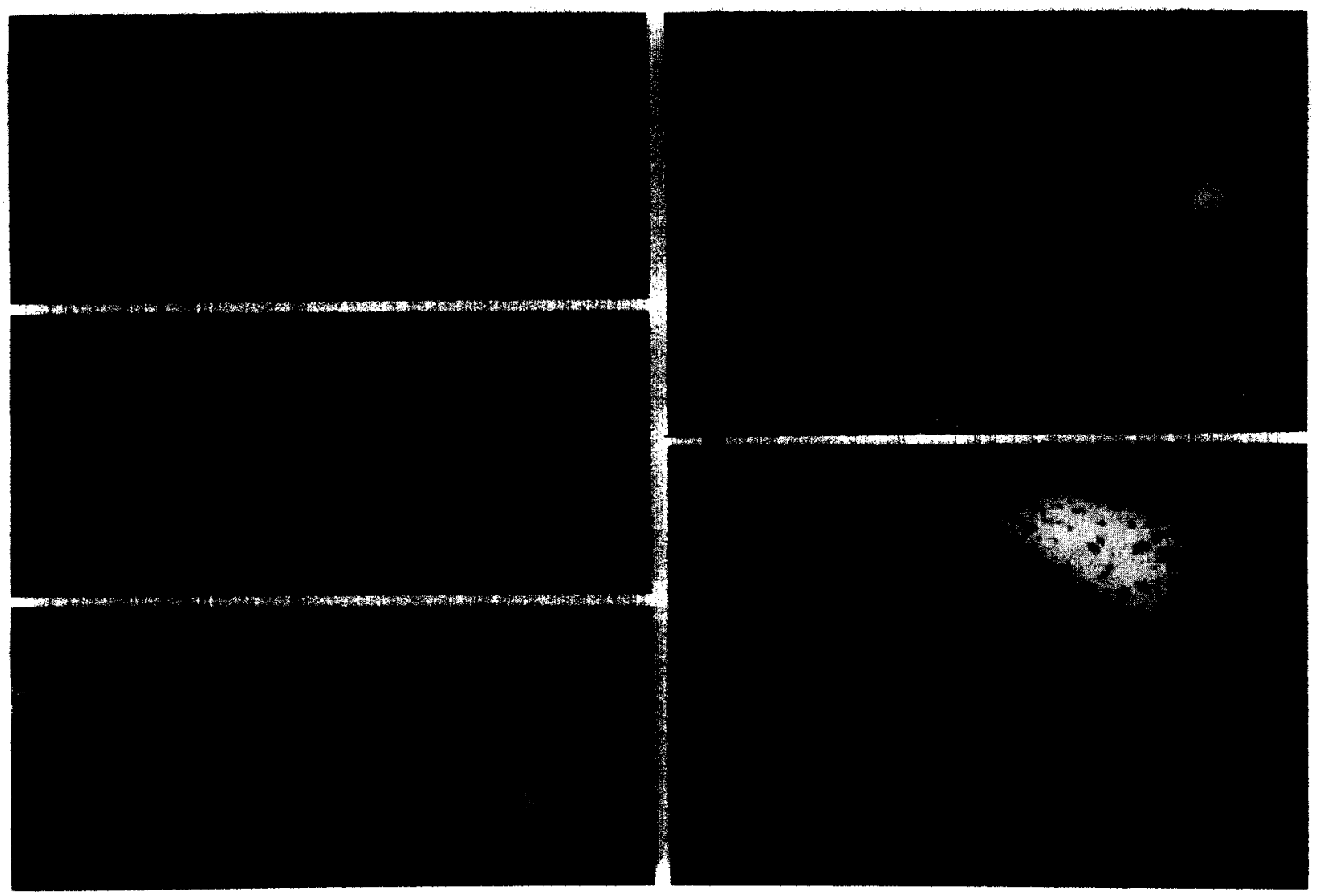

Fig. 3. a: dark-field photomicrograph at the level of the obex showing fascicles running between the trigeminal tract (left) and the solitary nucleus (right). $\times 56 . \mathrm{b}$ and $\mathrm{c}$ : dark-field photomicrographs of the solitary tract and nucleus to show the distribution of label. In $b$ HRP was injected into the tongue and in $c$ HRP was applied to the cut lingual-tonsilar branch of the glossopharyngeal nerve. Both sections are taken from approximately the same level in the brain. In b the solitary nucleus has been outlined with arrows. $\times 56$. $d$ : darkfield photomicrograph of a coronal brain section at the level of the area postrema. The dense label in the solitary nucleus is located in the interstitial nucleus. Labeled fascicles are seen coursing between the trigeminal tract and solitary nucleus. The labeled cells are hypoglossal motoneurons. $\times 35$. e: dark-field photomicrograph of the cluster of parasympathetic neurons situated at the most rostral level of the entering afferent glossopharyngeal fibers. The midline is toward the right. Efferent fibers are observed towards the bottom of the figure. Fibers travel towards the midline and form loops. Other fibers leave laterally, travelling towards the location of entering afferent fibers. $\times 126$.

(Fig. 2g). At this level numerous fascicles coursed between the spinal trigeminal tract and the solitary nucleus (Fig. 3a). Caudal to the area postrema the labeled fascicles in the spinal trigeminal tract rapidly diminished.

Afferent projections to the solitary nucleus descended in the solitary tract. Rostrally, at the level of entry of the fibers, terminal labeling was sparse. More caudally, the amount of terminal labeling increased and was located in both the lateral and dorsolateral medial solitary nucleus (Fig. 3b). The lateral label became more intense as the area postrema was reached and the medial label diminished. At the level of the area postrema there was an intense, terminal reaction product within the interstitial subnucleus of the solitary nucleus (Figs. $2 \mathrm{~g}, 3 \mathrm{~d}$ ). Caudal to the obex this intense label decreased and occasional fibers were observed crossing the midline.

Efferent fibers of the glossopharyngeal nerve exit the brainstem ventral to the point of entry of afferent fibers (Fig. 2a). The efferents coursed diagonally across the brainstem to their cells of origin in the nucleus ambiguus and a region just medial and ventral to the solitary nucleus. These latter cells formed a cluster (Fig. 3e) and extended from the caudal level of the dorsal cochlear nucleus, where they were few in number, to a region about $0.2-0.4 \mathrm{~mm}$ rostral to the most rostral afferent fibers of IX, where they 
were more numerous (approximately 30 cells per section). While the efferent processes of the majority of these cells were in the ventral root of the glossopharyngeal nerve, there were also a few processes in the mainly afferent, more dorsal root (Figs. 2a, 3e).

\section{Central projections of labeled glosspharyngeal nerve}

After applying HRP crystals to the cut cervical trunk of the glossopharyngeal nerve, similar brainstem structures were labeled as after papilla injection. The principal differences were that the labeling after nerve cut was unilateral and more intense (Fig. $3 c$ ), compared to labeling after papilla injection (Fig. $3 b)$. In addition the interconnections between the spinal trigeminal tract and the solitary nucleus were more numerous.

\section{DISCUSSION}

When horseradish peroxidase is injected into the region of the circumvallate gustatory papilla, it is transported centrally by afferent and efferent fibers of the glossopharyngeal nerve. The basic pattern of central projections after papilla injection is similar to that after labeling the cut IXth nerve. Essential elements of these projections include a distinct separation in the brainstem of afferent, dorsally located fibers and efferent, ventrally located fibers as described for the vagus nerve in rat ${ }^{9}$. Secondly, the afferent portion of the glossopharyngeal nerve has an extensive brainstem projection which contributes to the trigeminal system as well as to the solitary nucleus, and connections can be traced between the trigeminal and solitary components of the central projection. The pattern of terminal label within the solitary nucleus is complex. Rostrally it is located within both lateral and medial divisions of the solitary nucleus, while caudally it is intensely concentrated in the lateral division. This is especially evident at the level of the interstitial subnucleus of the solitary nucleus.

The extent of efferent labeling is less extensive. Motor cells are labeled in the nucleus ambiguus and in a more dorsally situated cluster that is juxtaposed to the solitary nucleus. Cells in the nucleus ambiguus presumably are muscle motoneurons whereas those situated near the solitary nucleus are probably the cells of origin of parasympathetic preganglionic fibers which form the inferior salivatory nucleus. The salivatory nucleus will be discussed later in detail.

These observations agree with recent studies of central projections of the rat glossopharyngeal nerve by Norgren and his co-workers ${ }^{3.6}$. In these studies, HRP applied to the cut lingual-tonsilar branch of the glossopharyngeal nerve resulted in an almost identical distribution of afferent and efferent projections in the brainstem as we found with papilla and nerve labling. This description for central projections of the glossopharyngeal nerve is similar also to results from classic experiments in the rat by Torvik ${ }^{21}$, who used degeneration methods, and to results in cat with HRP labeling ${ }^{16}$.

Having traced the central projections after both papilla injection and applying HRP to the cut central end of the glossopharyngeal nerve, some differences in the projections labeled by these two techniques are apparent. The label resulting from tongue injections is much less intense than that from the cut nerve. However, although labeling is less intense after papilla injection there is extensive labeling of the afferent and efferent fibers, indicating good transganglionic transport. Therefore it is likely that the intensity is reduced because only glossopharyngeal fibers innervating the circumvallate papilla are labeled, but not those innervating the foliate papillae on the lateral borders of the posterior tongue. In the rat there are approximately 230 taste buds in one foliate papilla and 350 in the circumvallate papilla ${ }^{12}$. All but 10 of the foliate taste buds (i.e. 220) are supplied by the glossopharyngeal nerve ${ }^{13}$ and $88 \%$ of the circumvallate taste buds are bilaterally innervated 5 . Thus, papilla injection should label fibers innervating 308 taste buds projecting to one side of the brain ( $88 \%$ of 350 ), whereas HRP on the cut glossopharyngeal nerve should label fibers supplying 528 taste buds (220 foliate +308 vallate). Papilla injection should, therefore, label the innervation to about $58 \%$ of taste buds labeled with nerve cut and result in less intense central reaction product.

\section{Incorporation of HRP after papilla injection}

HRP injected into the circumvallate papilla probably becomes incorporated into efferent fibers of the glossopharyngeal nerve at synaptic junctions between preganglionic fibers and the lingual autonomic ganglion cells, known as Remak's ganglia ${ }^{19}$. The label presumably enters afferent axons either at the 


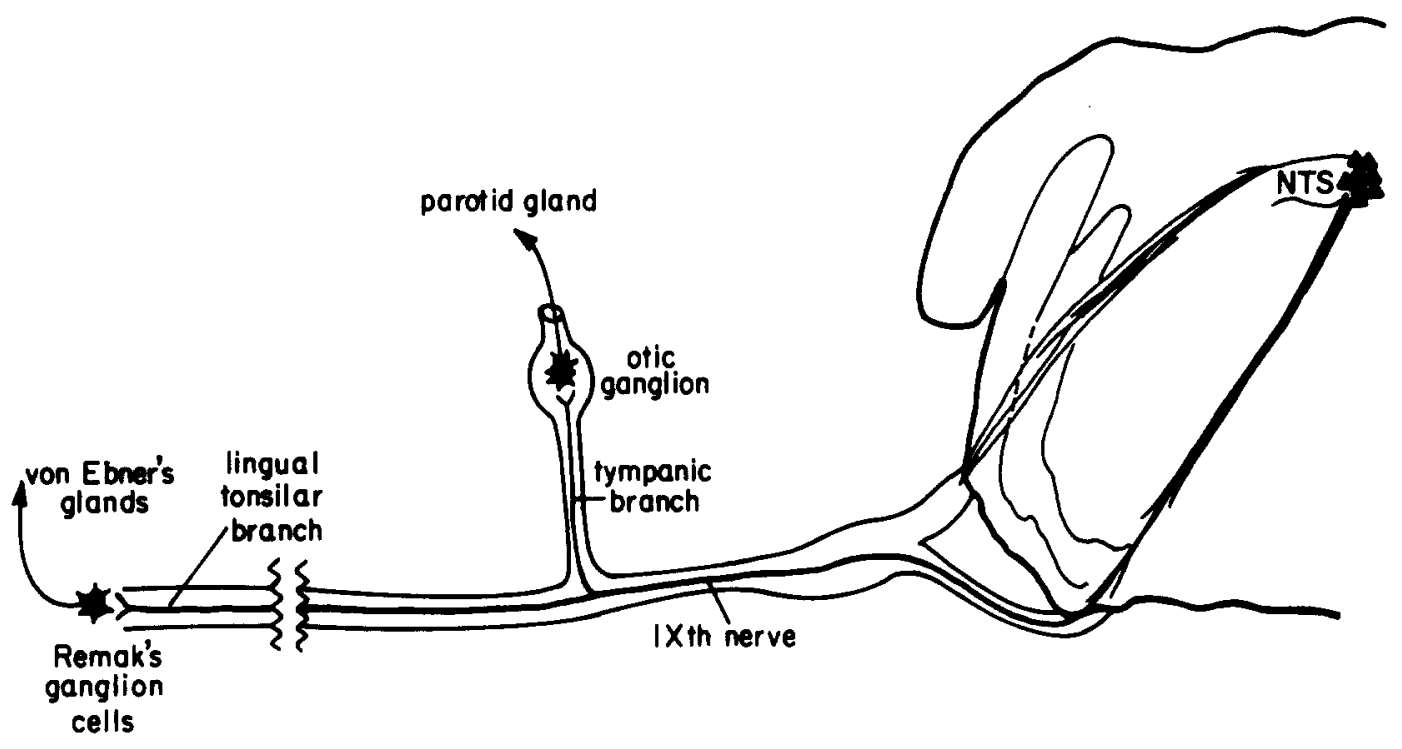

Fig. 4. Diagram of the parasympathetic pathways to the parotid and von Ebner's glands. Triangles in the brainstem represent cells of origin, in the inferior salivatory nucleus, of the parasympathetic preganglionic fibers to Remak's ganglion cells. Afferent fibers are diagrammed entering the brainstem at a more dorsal level, in a separate rootlet that projects to the nucleus of the solitary tract (NTS).

sensory terminals or as a result of trauma from the injection damage. Based on experiments by Brushart and Mesulam², who injected HRP into rat skin, it is probable that trauma at the injection site is not a critical factor in obtaining transganglionic transport of HRP. For example, when wheat germ agglutinin HRP was injected into rat skin, label was traced into the dorsal horn of the spinal cord. However, HRP alone in a non-conjugated form, or in combination with other agents known to enhance penetration, was not transported from the site of injection. The use of the lectin wheat germ agglutinin facilitated the uptake of HRP into the compartment of transport and was essential to obtain successful transport after skin injection; trauma alone apparently was not a key factor.

\section{Inferior salivatory nucleus and von Ebner's glands}

A discrete set of cells adjacent to the solitary nucleus has been described a number of times by various investigators who applied HRP to the cut, central end of the glossopharyngeal nerve ${ }^{3,14,20}$. These cells are parasympathetic neurons that usually are described as forming the inferior salivatory nucleus, and classically are thought to control secretion of the parotid salivary gland. However, as diagrammed in Fig. 4, parasympathetic, preganglionic fibers to the parotid gland travel in the tympanic branch of the glossopharyngeal nerve and synapse with postganglionic cells in the otic ganglion ${ }^{3}$. Injection of HRP into the circumvallate papilla should not label fibers that travel to the otic ganglion. In fact, a comparison of the central distribution of the otic ganglion connections $^{3}$, shows that the labeled cells begin at the rostral extent of the tongue papilla injection and the label extends for some distance more rostrally. It is more likely that the parasympathetic neurons which we label after papilla injection are preganglionic fibers that travel to Remak's ganglia in the posterior tongue. The postganglionic neurons are secretomotor to von Ebner's glands and vasodilator fibers ${ }^{4}$. Furthermore, Nomura and Mizuno ${ }^{15}$ concluded also that the cluster of neurons labeled near the solitary nucleus after applying HRP to the cat glossopharyngeal nerve send parasympathetic preganglionic fibers to the lingual ganglia. Therefore, the inferior salivatory nucleus apparently controls secretion of both the parotid and von Ebner's salivary glands.

Von Ebner's glands are intimately associated with taste buds in the circumvallate papilla (see Fig. 1). Their secretion drains into the trough surrounding the papilla, so that the immediate fluid environment of the taste buds is formed by the secretion from these glands. The glands presumably function to rinse the papilla troughs and thus, control access of stimuli to the taste receptors. It is likely, therefore, 
that there are interactions between taste receptors and von Ebner's glands. For example, taste afferent information could alter salivary composition and secretory rate, and the secretions produced could then alter afferent taste information. The location of the parasympathetic neurons innervating these glands just adjacent to the solitary nucleus in the medulla might facilitate neural interactions between afferent taste information and secretomotor efferent activity. At present there is little information on the characteristics of these interactions which are of importance in understanding the taste system.

\section{Conclusions}

It is clear from these results that it is possible to label the central projection of a taste papilla by injecting wheat germ agglutinin HRP into the papilla. In the rat, which has a single circumvallate papilla, the central distribution after papilla injection is similar to that produced by placing HRP on the cut branch of the glossopharyngeal nerve that supplies the posterior tongue. In animals with multiple circumvallate papillae, it should be possible to inject select papillae in the field and then determine the topographical projections of the papillae. In theory the injection tech-

\section{REFERENCES}

1 Arvidsson, J., Somatotopic organization of vibrissae afferents in the trigeminal sensory nuclei of the rat studied by transganglionic transport of HRP, J. Comp. Neurol., 211 (1982) 84-92.

2 Brushart, M. and Mesulam, M.-M., Transganglionic demonstration of central sensory projections from skin and muscle with HRP-lectin conjugates, Neurosci. Lett., 17 (1980) 1-6.

3 Contreras, R.J., Gomez, M.M. and Norgren, R., Central origins of cranial nerve parasympathetic neurons in the rat, J. Comp. Neurol., 190 (1980) 373-394.

4 Graziadei, P.P.C. and Monti Graziadei, G.A., Observations on the ultrastructure of ganglion cells in the circumvallate papilla of rat and mouse, Acta Anat., 100 (1978) 289-305.

5 Guth, L., Histological changes following partial denervation of the circumvallate papilla of the rat, Exp. Neurol., 8 (1963) 336-349.

6 Hamilton, R.B. and Norgren, R., Central projections of the gustatory nerves in the rat, J. Comp. Neurol., 222 (1984) 560-577.

7 Hess, D.T. and Schneider, G.E., Advantages of polarization microscopy in horseradish peroxidase neurohistochemistry, J. Histochem. Cytochem., 29 (1981) 1448-1450.

8 Hosoya, Y. and Sugiura, Y., The primary projection of the nique also would be applicable to mapping fungiform papilla projections. However, this might be more difficult since the papillae are small and not as densely innervated as the circumvallate. The usefulness of papilla injections for investigating topography in the taste system remains to be determined experimentally, but it promises to lead to new information on central connections of the gustatory sense.

Results from papilla injections in rat demonstrate also that cells of origin of the parasympathetic preganglionic fibers of Remak's ganglia are labeled in the inferior salivatory nucleus. Since the postganglionic fibers of Remak's ganglia are secretomotor to von Ebner's gland, it is apparent that the inferior salivatory nucleus regulates secretion of these lingual salivary glands.

\section{ACKNOWLEDGEMENTS}

This research was supported by N.I.H. Grants DE 05728 and DE 06825 and N.S.F. Grant BNS 8311497 to R.M.B. and C.M.M., N.S.F. Grant BNS81-20658 to H.P.K., and N.I.H. Fellowship MH08610 to C.A.B.

greater petrosal nerve to the solitary complex in the rat, revealed by transganglionic transport of horseradish peroxidase, Neurosci. Lett., 44 (1984) 13-17.

$9 \mathrm{Kalia}, \mathrm{M}$. and Sullivan, J.M., Brainstem projections of sensory and motor components of the vagus nerve in the rat, J. Comp. Neurol., 211 (1982) 248-264.

10 Krammer, E.B., Rath, T. and Lischka, M.F., Somatotopic organization of the hypoglossal nucleus: a HRP study in the rat, Brain Research, 170 (1979) 533-537.

11 Mesulam, M.-M., Principles of horseradish peroxidase neurohistochemistry and their applications for tracing neural pathways-axonal transport, enzyme histochemistry and light microscopic analysis. In M.-M., Mesulam (Ed.), Tracing Neural Connections with Horseradish Peroxidase, John Wiley, New York, 1982, pp. 1-151.

12 Miller, I.J., Gustatory receptors of the palate. In Y. Katsuki, M. Sato, S.F. Takagi and Y. Oomura (Eds.), Food Intake and Chemical Senses, University of Tokyo Press, Tokyo, 1977, pp. 173-185.

13 Miller, I.J., Gomez, M.M. and Lubarsky, E.H., Distribution of the facial nerve to taste receptors in the rat, Chem. Senses Flav., 3 (1978) 397-411.

14 Nicholson, J.E. and Severin, C.M., The superior and inferior salivatory nuclei in the rat, Neurosci. Lett., 21 (1981) 149-154.

15 Nomura, S. and Mizuno, N., Central distribution of afferent and efferent components of the chorda tympani in the 
cat as revealed by the horseradish peroxidase method, Brain Research, 214 (1981) 229-237.

16 Nomura, S. and Mizuno, N., Central distribution of afferent and efferent components of the glossopharyngeal nerve: an HRP study in the cat, Brain Research, 44 (1982) $1-13$.

17 Nomura, S. and Mizuno, N., Central distribution of efferent components in the greater petrosal nerve of the cat, Neurosci. Lett., 39 (1983) 11-14.

18 Nomura, S. and Mizuno, N., Central distribution of efferent and afferent components of the cervical branches of the vagus nerve: a HRP study in the cat, Anat. Embryol., 166 (1983) 1-18.

19 Remak, R., Über die Ganglien der Zunge bei Saugetieren und beim Menschen, Arch. Anat. Physiol. Wissensch., 17 (1852) 56-62.

20 Satomi, H., Yamamoto, T., Ise, H. and Takahashi, K., Identification of the inferior salivatory nucleus in the cat as studied by HRP bathings of the transected glossopharyngeal nerve root, Neurosci. Lett., 11 (1979) 259-264.

21 Torvik, A., Afferent connections to the sensory trigeminal nuclei, the nucleus of the solitary tract and adjacent structures. An experimental study in the rat, J. Comp. Neurol., 106 (1956) 51-141.

22 Whitehead, M.C. and Frank, M.E., Anatomy of the gustatory system in the hamster: central projections of the chorda tympani and the lingual nerves, J. Comp. Neurol., 220 (1983) 378-395. 